\title{
Radiation related complications after ruthenium plaque radiotherapy of uveal melanoma
}

Paula Summanen, Ilkka Immonen, Tero Kivelä, Petri Tommila, Jorma Heikkonen, Ahti Tarkkanen
Helsinki University Central Hospital, Helsinki, Finland

Department of Ophthalmology P Summanen

I Immonen

T Kivelä

P Tommila

A Tarkkanen

Department of Oncology

J Heikkonen

Correspondence to: Paula Summanen, MD, Department of Ophthalmology, Helsinki University Central Hospital Haartmaninkatu $4 \mathrm{C}$, FIN-00290 Helsinki

Finland.

Accepted for publication 24 May 1996

\begin{abstract}
Aims/background-To analyse radiation related complications and secondary enucleation after irradiation of malignant uveal melanoma with ruthenium-106 plaques.
\end{abstract}

Methods-A series of 100 consecutive eyes irradiated in 1981-91 was analysed using the life table method and the Cox proportional hazards model. The median apical and scleral tumour dose was 100 Gy (range 15-200 Gy) and $1000 \mathrm{~Gy}$ (range 200-1200 Gy), respectively. The median follow up time was 2.8 and 2.0 years (range 1 month to 10 years) for anterior and posterior segment complications, respectively.

Results-The 3 and 5 year probabilities of being without radiation cataract were $73 \%$ and $63 \%$, without neovascular glaucoma $91 \%$ and $81 \%$, without vitreous haemorrhage $83 \%$ and $74 \%$, without radiation maculopathy $85 \%$ and $70 \%$, and without radiation optic neuropathy $90 \%$ and $88 \%$, respectively. The risk of radiation cataract was highest with large tumour size (T1+T2 $v$ T3, p=0.0027; height $\leqslant 5 v>5 \mathrm{~mm}$, $\mathrm{p}=0.029$; largest basal diameter (LBD) $\leqslant 15 v>15 \mathrm{~mm}, p<0.0001$ ) and location of anterior tumour margin anterior $v$ posterior to the equator $(p=0.0003)$; the risk of neovascular glaucoma with large size (T1+T2 $v$ T3, p=0.039; LBD $\leqslant 15 \mathrm{~mm} v 15$ $\mathrm{mm}, \mathrm{p}=0.021$ ); and the risk of maculopathy and optic neuropathy with proximity of the posterior tumour margin to the fovea and the optic disc ( $\leqslant 1.5 v>1.5 \mathrm{~mm}$; $p=0.030$ and $p=0.0004$, respectively). In Cox's multivariate analysis the strongest risk indicator for radiation cataract ( $R R$ $1.5,95 \%$ CI 1.4-1.6) and vitreous haemorrhage (RR 1.6, 95\% CI 1.4-1.8) was the height of the tumour; for neovascular glaucoma the TNM class (RR 6.2, 95\% CI 2.7-13.8); for radiation maculopathy location of posterior tumour margin within 2 $\mathrm{mm}$ from the fovea (RR 3.4, 95\% CI 2.0-6.0); and for radiation optic neuropathy location of tumour margin within 1 DD of the optic disc (RR 6.1, 95\% CI $3.0-12.4)$. The 3 and 5 year probabilities of avoiding enucleation were $92 \%$ and $85 \%$, respectively. Ten eyes were enucleatedsix because of recurrent tumour growth, three because of treatment complications, and one because of mistakenly suspected extraocular growth.
Conclusion-The results suggest that the frequency of radiation related complications after ruthenium brachytherapy of uveal melanoma is acceptable, in particular as regard irradiation of small and medium sized tumours for which ruthenium therapy generally is recommended. (Br f Ophthalmol 1996;80:732-739)

The advent of conservative therapies ${ }^{1-5}$ has changed the treatment policy of malignant melanoma of the uvea and has made it possible to preserve the eye and some of the remaining sight for a considerable period of time. However, uveal melanomas are quite resistant to irradiation and the doses needed lead to radiation related complications that become increasingly common during the follow up..$^{6-9}$

Few studies have analysed the occurrence of radiation related complications by correcting statistically for unequal follow up times. ${ }^{11}$ Such reports are available for uveal melanomas irradiated with charged particles ${ }^{82-16}$ or with cobalt, ${ }^{17}$ iodine, ${ }^{1218}$ and ruthenium ${ }^{19}$ plaque brachytherapy. They focus on radiation cataract, ${ }^{8151718}$ neovascular glaucoma, ${ }^{812-14}$ vitreous haemorrhage, ${ }^{12}{ }^{18}$ radiation maculopathy, ${ }^{16}$ and ischaemic radiation optic neuropathy. ${ }^{19}$

We report the cumulative probabilities of remaining free from main radiation related complications in a series of 100 eyes of 100 patients treated because of malignant uveal melanoma with ruthenium-106 brachytherapy. We analyse their appearance in relation to the size and location of the tumour, ${ }^{246-622}$ both known to be risk indicators for loss of vision ${ }^{23}$ and tumour death. ${ }^{24}$ Such data on the frequency of complications should prove useful when planning the treatment and counselling the patient.

\section{Patients and methods}

Radiotherapy with ruthenium plaques began at the Department of Ophthalmology, Helsinki University Central Hospital, in March 1981. By February 1991, 100 eyes of 100 patients (41 men and 59 women; age range 22-84 years, median 59 years) with uveal melanoma had been treated with them. The patient selection criteria, local tumour relapse, metastasis, mortality, and visual outcome of these patients have been recently presented in detail. ${ }^{23}{ }^{24}$

PATIENTS AND THEIR FOLLOW UP

Before irradiation, all patients underwent a detailed ophthalmic evaluation including the 
Table 1 Characteristics of the 100 consecutive eyes with malignant uveal melanoma treated with ruthenium-106 brachytherapy

\begin{tabular}{|c|c|c|}
\hline Clinical characteristic & $N$ & $(\%)$ \\
\hline \multicolumn{3}{|l|}{ Anatomical location $(n=100)$} \\
\hline Ciliary body alone or with iris & 4 & (4) \\
\hline Ciliary body and choroid & 8 & (8) \\
\hline Choroid only & 88 & (88) \\
\hline \multicolumn{3}{|l|}{ TNM class $(n=100)^{25}$} \\
\hline $\mathrm{T} 1$ & 7 & (7) \\
\hline $\mathrm{T} 2$ & 37 & (37) \\
\hline T3 & 56 & (56) \\
\hline \multicolumn{3}{|l|}{ Height $(n=100)$} \\
\hline$\leqslant 3 \mathrm{~mm}$ & 11 & (11) \\
\hline$>3-5 \mathrm{~mm}$ & 35 & (35) \\
\hline$>5 \mathrm{~mm}$ & 54 & (54) \\
\hline \multicolumn{3}{|c|}{ Largest basal diameter (LBD) $(n=100)$} \\
\hline$\leqslant 10 \mathrm{~mm}$ & 44 & $(44)$ \\
\hline$>10-15 \mathrm{~mm}$ & 44 & (44) \\
\hline$>15 \mathrm{~mm}$ & 12 & (12) \\
\hline \multicolumn{3}{|l|}{ Location relative to equator $(n=100)$} \\
\hline Anterior & 5 & (5) \\
\hline Posterior & 62 & (62) \\
\hline Both sides & 33 & (33) \\
\hline \multicolumn{3}{|c|}{$\begin{array}{l}\text { Location relative to the optic disc and } \\
\text { fovea }(n=92)\end{array}$} \\
\hline$\leqslant 1 \mathrm{DD}$ of the disc & 23 & (25) \\
\hline$>1 \mathrm{DD}$ of the disc & 69 & (75) \\
\hline$\leqslant 1 \mathrm{DD}$ of the fovea & 48 & (52) \\
\hline$>1 \mathrm{DD}$ of the fovea & 44 & (48) \\
\hline
\end{tabular}

best corrected visual acuities, biomicroscopy, applanation tonometry, indirect ophthalmoscopy, gonioscopy, Goldmann three mirror lens examination, and B-scan ultrasonography. A fundus drawing was made. Photographs and fluorescein angiographs were technically possible to take from 97 and 79 tumours, respectively.

The height and largest basal diameter (LBD) of the tumour, its anatomical location and location in relation to the equator, and the TNM class ${ }^{25}$ were determined as described ${ }^{24}$ (Table 1). The median height of the tumour was $6 \mathrm{~mm}$ (range $1-15 \mathrm{~mm}$ ) and the median LBD $11 \mathrm{~mm}$ (range 4-25 mm). During the study period also large tumours $\left(\mathrm{T} 3^{25} ;>5 \mathrm{~mm}\right.$ in height or $>15 \mathrm{~mm}$ in the LBD) were initially accepted for brachytherapy with ruthenium plaques. $^{23}$ After iodine-125 plaques became available, ruthenium brachytherapy of large tumour was abandoned.

The distance from the posterior tumour margin to the fovea (range $0-17$, median 1.5 $\mathrm{mm}$ ) and to the rim of the optic disc (range $0-14$, median $3 \mathrm{~mm}$ ) were measured by ophthalmoscopy, and confirmed from fundus photographs. They were recordable for all tumours except five located entirely anterior to the equator and for three obscured by hazy media (Table 1).

During surgery, the tumour was located with the help of trans-scleral illumination with a fibreoptic light, indirect ophthalmoscopy with scleral indentation, or both. The margins of the tumour were marked on the sclera with diathermy. Indentation around the plaque was used to confirm the positioning of very posterior plaques. The median apical and scleral tumour doses were 100 Gy (range 15-200 Gy) and $1000 \mathrm{~Gy}$ (range 200-1200 Gy), respectively, delivered within 1 to 23 days (median 5.5 days).

After irradiation, follow up visits took place $1,3,6$, and 12 months after irradiation, then twice a year until the fourth postoperative year, and annually thereafter. At every follow up examination a careful clinical evaluation, ultrasonography, and fundus photographs were repeated, while the annual examination additionally included fluorescein angiography.

Follow up data to February 1992 were available for all patients. The median follow up time for development of anterior segment complications was 2.8 years. It was 2.0 years for development of posterior segment complications because of media opacities that increased during the follow up (range for both, 1 month to 10 years). The follow up time was less than 1 year for seven eyes, six of which were enucleated during this time. All except four patients were followed up at our hospital. Their latest follow up data and data from five patients who developed metastases were obtained from other hospitals.

The dates when the various radiation related complications developed were recorded. Generally this had occurred between two follow up examinations, and the eye was censored from analysis at the midpoint of that interval. ${ }^{22}{ }^{26}$ For cataract, rubeosis of the iris, and neovascular glaucoma follow up was taken to end at the latest ophthalmic examination, while for posterior segment complications (vitreous haemorrhage, maculopathy, and optic neuropathy) it ended at the last examination when the posterior segment was still assessable. Two eyes treated for local relapse with an iodine plaque ${ }^{24}$ were censored on the day preceding the retreatment. Eight eyes irradiated twice with ruthenium were not censored from the analysis. Owing to the low number of these eyes, the influence of repeated brachytherapy could not be analysed separately. The date and the cause of enucleation were recorded.

\section{DEFINITIONS OF RADIATION RELATED COMPLICATIONS}

The appearance of either posterior cortical or subcapsular cataract or a sectoral lens opacity $^{1227}$ that developed or clearly progressed asymmetrically after treatment was interpreted as radiation cataract. It was assessable in 99 eyes; the remaining eye was pseudophakic. Iris rubeosis was defined as new blood vessels over the iris stroma, the chamber angle, or both; and neovascular glaucoma as a rise in intraocular pressure above $23 \mathrm{~mm} \mathrm{Hg}$ in an eye with iris neovascularisation and a previously normal pressure. They could be assessed in all eyes.

The occurrence of postoperative vitreous haemorrhage could be assessed in 96 eyes; three eyes with a dense haemorrhage present before therapy, and one with a mature cataract at the first postoperative examination were non-assessable. Radiation maculopathy ${ }^{28}$ was defined as retinal vascular changes such as microaneurysms, haemorrhages, microinfarcts, and lipid exudates seen clinically in the macular area (diameter 1.5 DD). It could be assessed in 85 eyes; in addition to the four eyes mentioned, three were excluded owing to the presence of microaneurysms before therapy, six because of exudative retinal detachment involving the macula, and two because of a 
Table 2 Results of Cox proportional hazards multivariate survival analysis for main radiation related complications after ruthenium-106 brachytherapy with estimated rate ratios (RR) and their $95 \%$ confidence intevals (CI)

\begin{tabular}{|c|c|c|c|c|c|c|c|}
\hline \multirow[b]{2}{*}{ Clinical variable } & \multirow[b]{2}{*}{ Comparison } & \multicolumn{6}{|l|}{ Complication } \\
\hline & & $\begin{array}{l}\text { Cataract } \\
R R(C I)\end{array}$ & $\begin{array}{l}\text { Iris rubeosis } \\
R R(C I)\end{array}$ & $\begin{array}{l}\text { Neovascular } \\
\text { glaucoma } \\
R R(C I)\end{array}$ & $\begin{array}{l}\text { Vitreous } \\
\text { haemorrhage } \\
R R(C I)\end{array}$ & $\begin{array}{l}\text { Maculopathy } \\
R R(C I)\end{array}$ & $\begin{array}{l}\text { Optic neuropathy } \\
R R(C I)\end{array}$ \\
\hline $\begin{array}{l}\text { Height of tumour } \\
\text { Largest basal diameter (LBD) } \\
\text { Size of tumour } \\
\text { Distance from fovea } \\
\text { Distance from optic disc rim }\end{array}$ & $\begin{array}{l}\leqslant 5 v>5 \mathrm{~mm} \\
\leqslant 15 v>15 \mathrm{~mm} \\
\mathrm{~T} 1+\mathrm{T} 2 v \mathrm{~T} 3 \\
\leqslant 2 v>2 \mathrm{~mm} \\
\leqslant 1 v>1 \mathrm{DD} \dagger\end{array}$ & $\begin{array}{l}1.5(1.4-1.6) \\
1.1(1.1-1.2)\end{array}$ & $1.2(1.1-1.2)$ & $6.2(2.7-13.8)$ & $1.6(1.4-1.8)$ & $3.4(2.0-6.0)$ & $6.1(3.0-12.4)$ \\
\hline
\end{tabular}

* Tumour size according to the TNM classification. ${ }^{25}$

$\mathrm{t} \mathrm{DD}=$ disc diameter.

large tumour obscuring the macula. Macular pucker and diffuse or cystoid oedema without other changes were not classified as radiation maculopathy.

Development of anterior ischaemic optic neuropathy caused by vascular occlusion and seen as oedema, haemorrhages, microinfarcts, and narrowing of optic disc vessels ${ }^{29}$ could be evaluated in 94 eyes. The four eyes with cloudy media already mentioned, and two other eyes in which the tumour covered the disc totally were excluded from the analysis.

\section{DATA ANALYSIS}

The data were stored in a mainframe computer and analysed with the BMDP software. ${ }^{30}$ The range and median were calculated for the quantitative variables. Cumulative proportions (SE) of eyes remaining free of various radiation related complications were calculated using the life table method ${ }^{10}{ }^{110}$ and plotted until fewer than five patients remained in any particular arm to avoid instability of the tail of the curve. ${ }^{26}$

Univariate analysis in terms of size and location of the tumour relative to the equator, to the fovea, and to the rim of the optic nerve head was done by the Mantel-Cox test. ${ }^{30}$ The size of the tumour was analysed both according to the TNM class ${ }^{25}$ and the height and LBD of the tumour. Small and medium sized tumours $(\mathrm{T} 1+\mathrm{T} 2)$ were combined and compared with large ones (T3). Cutoff points for height and LBD were $5 \mathrm{~mm}$ and $15 \mathrm{~mm}$, respectively. Eyes with a tumour at least in part anterior to the equator with or without ciliary body involvement were compared with those in which it was entirely posterior to the equator.

The effect of tumour size was assessed for all complications, the location relative to the equator for all anterior segment complications and vitreous haemorrhage, and the proximity of the posterior tumour margin to the fovea and the optic disc for radiation maculopathy and optic neuropathy, provided that the tumour was located at least in part posterior to the equator. A p value less than 0.05 was considered significant.

Cox proportional hazards model ${ }^{30}$ was used to identify independent predictors for development of radiation related complications. In addition to the variables mentioned, involvement of ciliary body versus choroid only was analysed. The results are given as the rate ratio $(\mathrm{RR})$ and its $95 \%$ confidence interval (CI).

\section{Results}

Radiation related complications developed in 50 of the 100 eyes after ruthenium-106 brachytherapy during a median follow up time of 2.8 and 2.0 years for anterior and posterior segment complications, respectively. Out of these 50 eyes, 21 eyes (42\%) had developed one, 19 eyes (38\%) two, and 10 eyes $(20 \%)$ three or more complications by latest follow up examination.

\section{ANTERIOR SEGMENT COMPLICATIONS}

\section{Radiation cataract}

New lens opacities developed in 26 of 99 treated eyes $(26 \%) 0.1$ to 10.1 years (median 1.3) after irradiation. The overall cumulative 2 , 3 , and 5 year probabilities of an eye remaining free from radiation cataract were $79 \%, 73 \%$, and $63 \%$, respectively (Fig 1A). It was diagnosed significantly more frequently in eyes harbouring a large tumour, whether defined by TNM $\operatorname{class}^{25}(\mathrm{~T} 1+\mathrm{T} 2$ v T3; $\mathrm{p}=0.0027$, Mantel-Cox, difference between corresponding curves, Fig 1B), height ( $\leqslant 5 \mathrm{~mm} v>5 \mathrm{~mm}$; $\mathrm{p}=0.029$, Fig $1 \mathrm{C}$ ), or largest basal diameter (LBD) of the tumour ( $\leqslant 15 \mathrm{~mm} v>15 \mathrm{~mm}$; $\mathrm{p}<0.0001$, Fig 1D), and when the tumour was located at least in part anterior to the equator rather than entirely posterior to it $(p=0.0003$, Fig 1E). In Cox proportional hazards multivariate analysis, height of the tumour greater than $5 \mathrm{~mm}$ was the strongest predictor of developing a radiation cataract (RR 1.5, $95 \%$ CI 1.4-1.6; $\mathrm{p}<0.0001$, Table 2).

\section{Iris rubeosis}

Neovascularisation of the iris was detected in 12 of 100 eyes (12\%) 0.5 to 5.2 years (median 2.4) after irradiation. The overall cumulative 2 , 3 , and 5 year probabilities of an eye remaining free from iris rubeosis were $95 \%, 88 \%$, and $80 \%$, respectively (Fig $2 \mathrm{~A}$ ). Both large tumour size as defined by the TNM class (T1+T2 $v$ T3; p=0.066, Mantel-Cox, Fig 2B) and LBD $(\leqslant 15 \mathrm{~mm} v>15 \mathrm{~mm} ; \mathrm{p}=0.021$, Fig $2 \mathrm{C})$, and location of the tumour at least partially anterior to the equator $(p=0.054$, Fig $2 D)$ increased the probability of iris rubeosis, but only LBD reached statistical significance. Likewise, in Cox multivariate analysis, only a LBD of more than $15 \mathrm{~mm}$ significantly increased the risk of rubeosis (RR $1.2,95 \%$ CI $1.1-1.2, \mathrm{p}=0.012$, Table 2). 
Neovascular glaucoma

Glaucoma eventually developed in 10 of the 12 eyes $(85 \%)$ with iris neovascularisation $0.5-5.6$ years (median 2.6) after the irradiation. In six of these 10 eyes, exudative retinal detachment persisted at the latest examination. The cumulative 2,3 , and 5 year probabilities of an eye remaining free from neovascular glaucoma were $96 \%, 91 \%$, and $81 \%$, respectively (Fig 3A). Large tumour size as defined by the TNM class $(\mathrm{T} 1+\mathrm{T} 2 v \mathrm{~T} 3 ; \mathrm{p}=0.039$, Mantel-Cox, Fig 3B) and LBD ( $\leqslant 15 \mathrm{~mm} v>15 \mathrm{~mm} ; \mathrm{p}=0.021$, Fig 3C) was associated with a significantly increased probability of glaucoma, whereas the location of the tumour relative to the equator was not statistically significant (Fig 3D). In Cox multivariate analysis, TNM class $\mathrm{T} 3$ was a
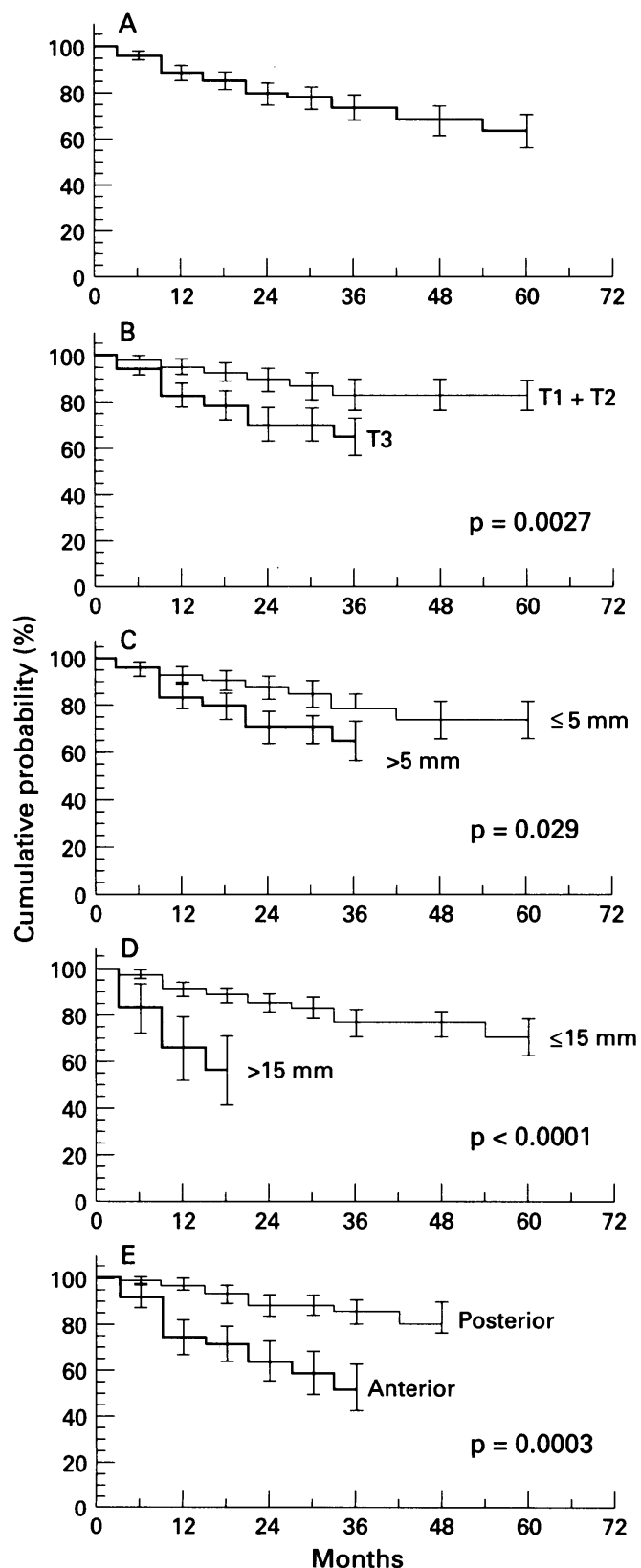

Figure 1 Cumulative probabilities (SE) of remaining free from radiation cataract after ruthenium-106 brachytherapy for malignant uveal melanoma for all 99 eyes included in the analysis $(A)$. Large size of the tumour as defined by (B) its TNM class (T1 and T2 v T3), (C) its height ( $\leqslant 5$ $m \mathrm{~mm}>5 \mathrm{~mm}$ ), and (D) its largest basal diameter ( $\leqslant 15$ $\mathrm{mm} v>15 \mathrm{~mm}$ ), as well as location of the anterior tumour margin anterior to the equator $(E)$ were associated with a significantly higher risk of radiation cataract. strong risk indicator for neovascular glaucoma (RR 6.2, 95\% CI 2.7-13.8, p= 0.012, Table 2).

\section{POSTERIOR SEGMENT COMPLICATIONS}

\section{Vitreous haemorrhage}

New vitreous haemorrhage occurred in 14 of 96 eyes (15\%) (including two of four eyes with bleeding already before therapy) 1.0 week to 4.1 years (median 1.4) after irradiation. The overall cumulative 2,3 , and 5 year probabilities of an eye remaining free from vitreous haemorrhage were $91 \%, 83 \%$, and $74 \%$, respectively (Fig 4A). Bleeding tended to occur more often in eyes with a large tumour as defined by the TNM class (Fig 4B), height (Fig 4C) and LBD, but these associations did not reach statistical significance. Likewise, location of the anterior tumour margin relative to the equator did not affect the risk for vitreous haemorrhage. In Cox multivariate analysis, tumour height of more than $5 \mathrm{~mm}$ was the only significant predictor of bleeding (RR 1.6, $95 \% \mathrm{CI}$ 1.4-1.8, $\mathrm{p}=0.001$, Table 2).

Two eyes were vitrectomised because of unresorbable haemorrhage. Both of them lost light perception and were eventually enucle-
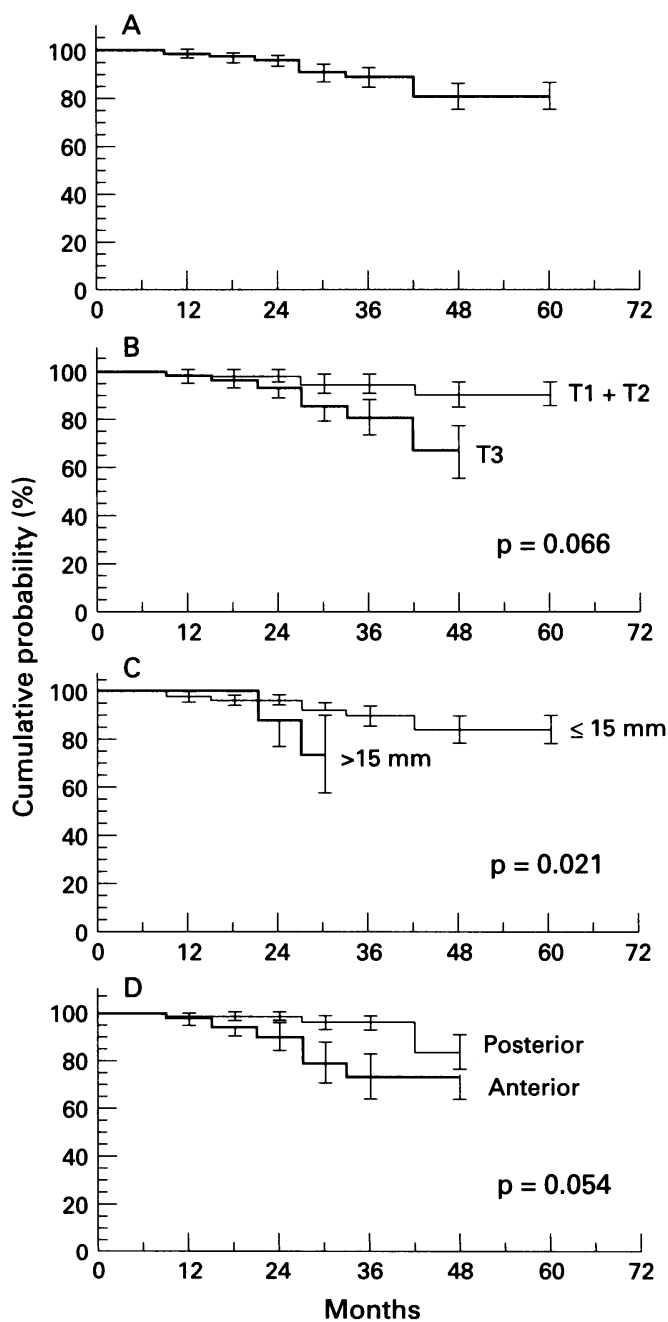

Figure 2 Cumulative probabilities (SE) of remaining free from rubeosis of the iris after ruthenium-106 brachytherapy for malignant uveal melanoma for all 100 eyes included in the analysis (A). Large size of the tumour as defined by (B) its TNM class (T1 and T2 v T3) and (C) its largest basal diameter ( $\leqslant 15 \mathrm{~mm} v>15 \mathrm{~mm})$, as well as location of the anterior tumour margin anterior to the equator (D) were associated with a tendency to a higher risk of rubeosis of the iris. 
ated, the first for cosmetic reasons and the other because of an indolent corneal ulcer secondary to neovascular glaucoma.

\section{Radiation maculopathy}

Radiation maculopathy was detected in 20 of the 85 eyes $(24 \%) 0.2$ to 4.3 years (median 1.8) after irradiation. The overall cumulative 2 and 3 year probabilities of an eye remaining free from maculopathy were $85 \%$ and $70 \%$, respectively (Fig 5A). The development of maculopathy was affected by the proximity of the posterior tumour margin to the fovea. A distance of more than $1.5 \mathrm{~mm}$ (1 DD) significantly reduced the risk of radiation maculopathy ( $p=0.030$, Mantel-Cox, Fig 5B). The 2 and 3 year probabilities of an eye being free from maculopathy were $74 \%$ and $53 \%$ when the tumour was within 1 DD of the fovea compared with $94 \%$ and $83 \%$ when it was located further than $1 \mathrm{DD}$, respectively. In Cox multivariate analysis, location of the posterior tumour margin within $2 \mathrm{~mm}$ (1.5 DD) from the fovea increased the risk of maculopathy by a factor of $3.4(95 \%$ CI $2.0-6.0, p=0.015$,
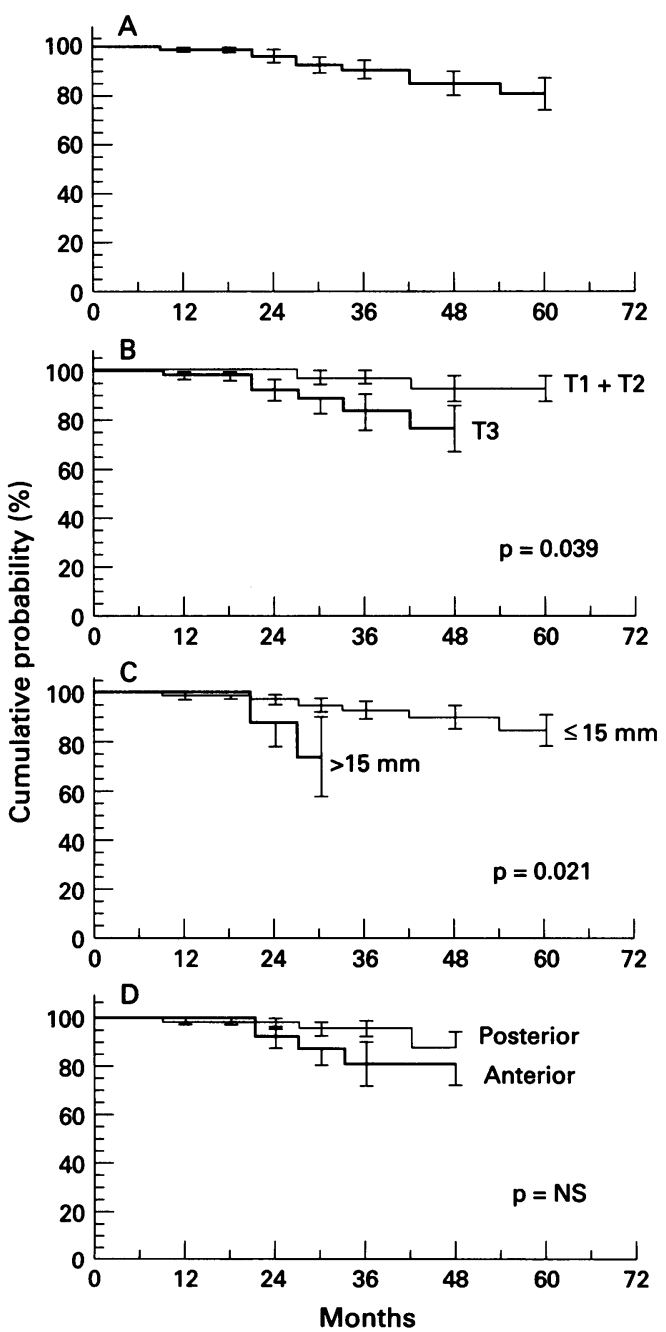

Figure 3 Cumulative probabilities (SE) of remaining free from neovascular glaucoma after ruthenium-106 brachytherapy for malignant uveal melanoma for all 100 eyes included in the analysis (A). Large size of the tumour as defined by (B) its TNM class (T1 and T2 $v T 3$ ) and (C) its largest basal diameter ( $\leqslant 15 \mathrm{~mm} v>15 \mathrm{~mm}$ ), unlike the location of the anterior tumour margin anterior to the equator (D) were associated with a significantly higher risk of neovascular glaucoma.
Table 2). Large tumour size was not associated with radiation maculopathy in either analysis.

Radiation optic neuropathy

Radiation induced ischaemic optic neuropathy was detected in nine of 94 eyes $(10 \%) 0.5$ to 3.8 years (median 1.6) after irradiation. The cumulative 2 and 3 year probabilities of an eye being free from radiation optic neuropathy were $90 \%$ and $88 \%$, respectively (Fig $6 \mathrm{~A}$ ). The
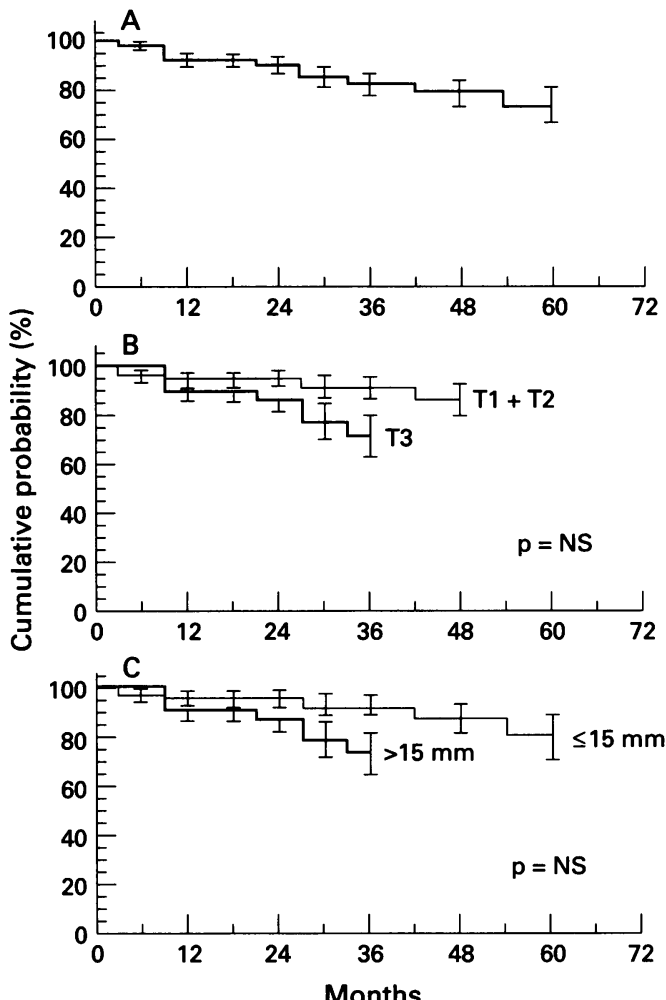

Figure 4 Cumulative probabilities (SE) of remaining free from vitreous haemorrhage after ruthenium-106 brachytherapy for malignant uveal melanoma for all 94 eyes included in the analysis $(A)$. Large size of the tumour as defined by (B) its TNM class (T1 and T2 $v$ T3) and (C) its height ( $\leqslant 5 \mathrm{~mm} v>5 \mathrm{~mm}$ ) were associated with a tendency to a higher risk of vitreous haemorrhage.

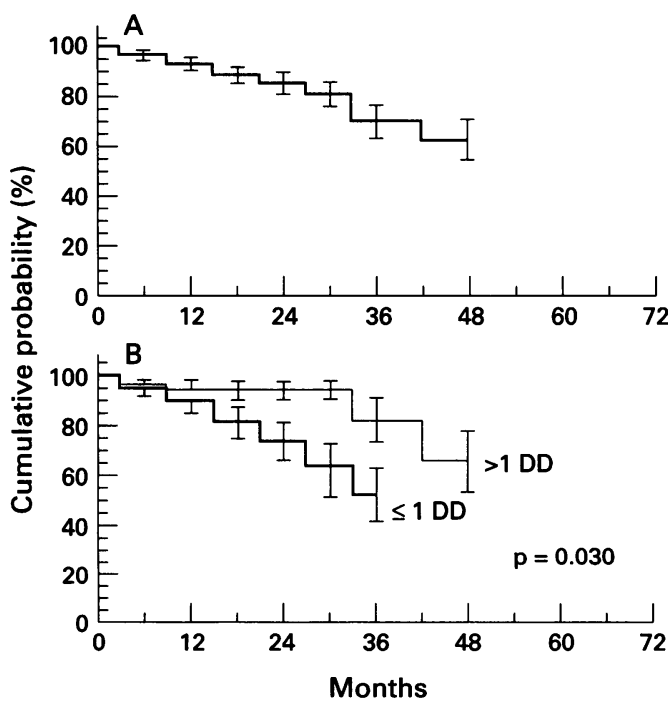

Figure 5 Cumulative probabilities (SE) of remaining free from radiation maculopathy after ruthenium-106 brachytherapy for malignant uveal melanoma for all 85 eyes included in the analysis $(A)$. Proximity of the posterior tumour margin to the fovea ( $\leqslant 1 D D v>1 D D$ from it) was associated with a significantly higher risk of radiation maculopathy (B). 
Table 3 Comparison of clinical characteristics and cumulative 2, 3, and 5 year probabilities of radiation related complications in various series correcting for the unequal follow up times with statistical methods

\begin{tabular}{|c|c|c|c|c|c|c|c|c|}
\hline \multirow[b]{2}{*}{ Reference } & \multicolumn{3}{|c|}{ Series characteristics } & \multicolumn{5}{|c|}{2,3 , and 5 year cumulative probabilities of radiation related complications } \\
\hline & $\begin{array}{l}\text { Type of } \\
\text { irradiation * }\end{array}$ & $\begin{array}{l}\text { Large sizet } \\
(\%)\end{array}$ & $\begin{array}{l}\text { Anterior } \\
\text { location } \neq \\
(\%)\end{array}$ & $\begin{array}{l}\text { Cataract } \\
(\%)\end{array}$ & $\begin{array}{l}\text { Neovascular } \\
\text { glaucoma } \\
(\%)\end{array}$ & $\begin{array}{l}\text { Vitreous } \\
\text { haemorrhage } \\
(\%)\end{array}$ & $\begin{array}{l}\text { Maculopathy } \\
(\%)\end{array}$ & $\begin{array}{l}\text { Optic } \\
\text { neuropathy } \\
(\%)\end{array}$ \\
\hline Char $(1989)^{12}$ & $\begin{array}{l}\mathrm{He} \\
\mathrm{I}\end{array}$ & & & & $\begin{array}{l}20,27,- \\
6,-,-\end{array}$ & $\begin{array}{l}7,9,15 \\
17,24,28\end{array}$ & & \\
\hline Char $(1990)^{8}$ & $\mathrm{He}$ & 62 & 59 & $9,27,45$ & $23,30,33$ & & & \\
\hline Decker $(1990)^{13}$ & $\mathrm{He}$ & & 100 & & $40,43,43$ & & & \\
\hline Lindstadt $(1990)^{14}$ & $\mathrm{He}$ & 66 & 22 & & $26,32,36$ & & & \\
\hline Guyer $(1992)^{16}$ & $\mathrm{~Pb}$ & 0 & 0 & & & & $55,69,78 \rrbracket$ & \\
\hline Kleineidam (1993) ${ }^{17}$ & Co & & 62 & $21,35,57$ & & & & \\
\hline Quivey $(1993)^{18}$ & I & 53 & 36 & $4,14,25$ & & $15,22,29$ & & \\
\hline Lommatzsch $(1994)^{19}$ & $\mathbf{R u}$ & & 0 & & & & & $\begin{array}{l}40,55,66^{\star \star} \\
7,10,23 \mathrm{tt}\end{array}$ \\
\hline Present series & Ru & 56 & 38 & $21,27,37$ & $4,11,21$ & $10,18,27$ & $15,30,-$ & $10,12,-$ \\
\hline
\end{tabular}

$\star \mathrm{Ru}=$ ruthenium plaque, $\mathrm{I}=$ iodine plaque, $\mathrm{Co}=$ cobalt plaque, $\mathrm{He}=$ helium ion, $\mathrm{Pb}=$ proton beam irradiation.

† More than $5 \mathrm{~mm}$ in height or more than $15 \mathrm{~mm}$ in largest basal diameter, or T3 in TNM classification.

$\ddagger$ At least in part anterior to the equator with or without ciliary body involvement.

$\S$ Intraretinal haemorrhages without other changes included.

$\star \star$ Partial optic atrophy.

†† Complete optic atrophy.

proximity of the posterior tumour margin to the rim of the optic disc highly significantly predicted the appearance of radiation optic neuropathy ( $\leqslant 1$ DD $v>1$ DD; $\mathrm{p}=0.0004$, Mantel-Cox, Fig 6B). It never developed when the distance was at least $4 \mathrm{~mm}(2.5 \mathrm{DD})$. In Cox multivariate analysis, location of the posterior tumour margin within $1 \mathrm{DD}$ increased the risk of radiation optic neuropathy by a factor of $6.1 \quad(95 \%$ CI $3.0-12.4$, $\mathrm{p}=0.0082$, Table 2). Optic neuropathy was not associated with large tumour size in either analysis.

\section{SECONDARY ENUCLEATION}

Ten eyes $(10 \%)$ were enucleated after a median follow up period of 10 months (range 1 month to 6.9 years) from irradiation. The cause of enucleation was recurrent tumour growth in six patients, a painful and blind eye with neovascular glaucoma with or without persistent exudative retinal detachment in three

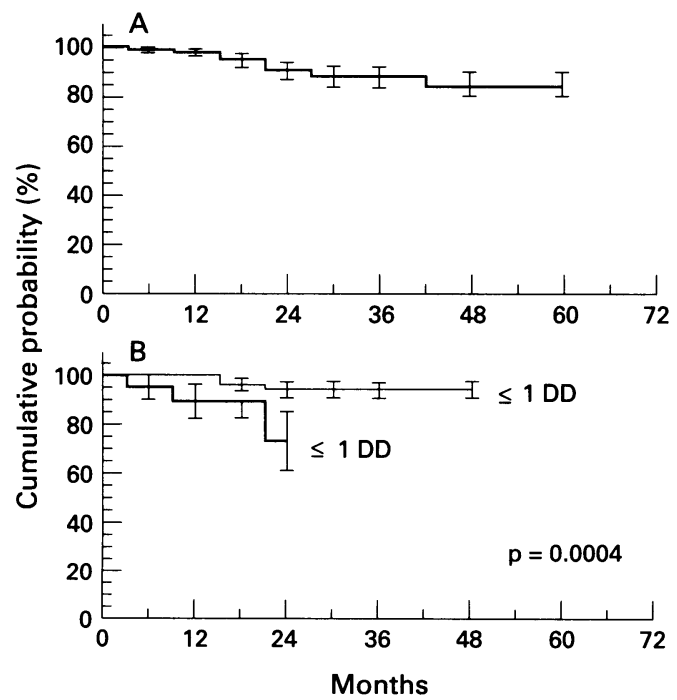

Figure 6 Cumulative probabilities (SE) of remaining free from radiation optic neuropathy after ruthenium-106 brachytherapy for malignant uveal melanoma for all 94 eyes included in the analysis $(A)$. Proximity of the posterior tumour margin to the rim of the optic disc $(\leqslant 1 D D v>1$ $D D$ from it) was associated with a significantly higher risk of radiation optic neuropathy $(B)$. patients, and suspected extrascleral tumour growth not proved by histopathological examination in one patient.

The cumulative 2,3 , and 5 year probabilities of escaping enucleation were $92 \%, 92 \%$, and $85 \%$, respectively (Fig 7A). Eyes with a large tumour were enucleated significantly more often than those with a small to medium sized tumour (T1+T2 $v \mathrm{~T} 3 ; \mathrm{p}=0.0083$, MantelCox, Fig 7B), the 2 and 3 year probabilities being $2 \%$ for small to medium sized and $13 \%$ for large tumours, respectively. The location of the tumour in relation to the optic disc or fovea was not statistically associated with risk of enucleation.

\section{Discussion}

In principle, our results might be compared with the nine previous series of malignant uveal melanoma in which unequal follow up times have been statistically corrected for. ${ }^{812-19}$ In practice, it is important to take into account differences in study populations, inclusion cri-

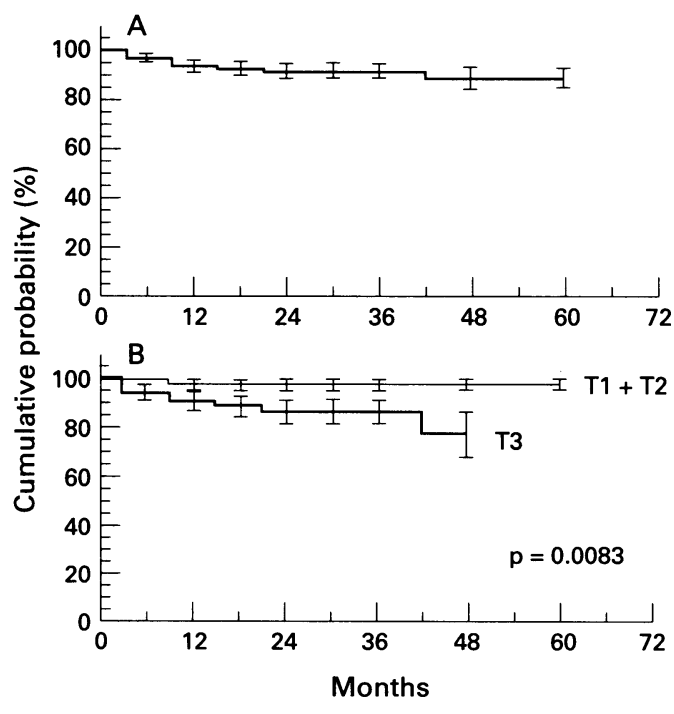

Figure 7. Cumulative probabilities (SE) of eyes escaping enucleation after ruthenium-106 brachytherapy for malignant uveal melanoma $(A)$ in the entire series of 100 treated eyes (A). Large tumour size $(T 1+T 2 v T 3)$ was associated with a significantly higher risk of enucleation (B). 
teria, and definitions of complications when making such comparisons. The proportion of large tumours in most of them was quite homogeneous, ${ }^{812-14} 1718$ varying from $53 \%$ to $66 \%$, whereas that of eyes with a tumour at least in part anterior to the equator varied from $32 \%$ to $62 \%$ (Table 3). Three particular series included only tumours that were small to medium sized, ${ }^{14}$ involved the ciliary body, ${ }^{13}$ or were juxtapapillary. ${ }^{19}$

In our series, cataract was the most frequent radiation related complication, the cumulative 2,3 , and 5 year probabilities being $21 \%, 27 \%$, and $37 \%$, respectively. In line with previous data, ${ }^{5917}$ the risk of developing a radiation cataract was higher when the tumour was large. In a series with quite similar proportions of large and anteriorly located tumours treated with iodine plaques these figures were lower, being $4 \%, 14 \%$, and $25 \%$, respectively. ${ }^{18}$ This may be due to the fact that 'significant' radiation cataracts only ${ }^{18}$ rather than all lens opacities $^{1227}$ were included in their analysis.

Neovascular glaucoma developed in 10 of 12 eyes $(85 \%)$ with iris rubeosis in our series, the cumulative 2,3 , and 5 year probabilities being $4 \%, 11 \%$, and $21 \%$, respectively. Persistent retinal detachment in six of them $(60 \%)$ may have been a contributing factor. Rubeotic glaucoma has been particularly common in helium ion series that have included many anteriorly located tumours (Table 3). In line with previous series, ${ }^{5}{ }^{12-14}$ the size rather than the location of the tumour was associated with development of neovascular glaucoma in univariate analysis. In Cox multivariate analysis, however, the TNM class turned out to be the best risk indicator for appearance of rubeotic glaucoma (T3; $R R \quad 6.2,95 \%$ CI 2.7-13.8) in our series. Interestingly, the TNM class takes into account the involvement of the ciliary body, because class T3 includes all choroidal tumours extending to the ciliary body regardless of their size. ${ }^{25}$

In the present series, the cumulative 2,3 , and 5 year probabilities of vitreous haemorrhage were $10 \%, 18 \%$, and $27 \%$, roughly equalling the corresponding figures of $15 \%$, $22 \%$ and $29 \%$, respectively, after iodine brachytherapy. ${ }^{18}$ As mentioned, this may be due to the similarity of tumours in these two studies. Large tumour size ${ }^{5}$ was not significantly associated with vitreous haemorrhage in our series, whereas height of the tumour was significant in Cox multivariate analysis ( $R R$ $1.6,95 \%$ CI 1.4-1.8). Vitreous haemorrhage after radiotherapy of uveal melanoma often clears spontaneously. ${ }^{4518}$ A need to operate may indicate poor prognosis. Both operated eyes in our series went blind.

Radiation maculopathy competed with cataract as the leading radiation related complication in our series, with cumulative 2 and 3 year probabilities of $15 \%$ and $30 \%$, respectively. Optic neuropathy, on the other hand, was relatively uncommon with corresponding frequencies of $10 \%$ and $12 \%$, respectively. Their frequencies were expectedly high, since $48 \%$ and $23 \%$ of the tumours were located within 1 $\mathrm{DD}$ of the fovea and the rim of the optic disc, respectively. In such eyes, these complications are common. ${ }^{672022} \mathrm{~A}$ distance greater than 1.5 $\mathrm{mm}(1 \mathrm{DD})$ from the fovea in univariate analysis and more than $2 \mathrm{~mm}(1.5 \mathrm{DD})$ in multivariate analysis predicted a significantly reduced risk of radiation maculopathy. This complication was a frequent cause of losing reading vision in the irradiated eye, ${ }^{23}$ whereas rubeotic glaucoma and enucleation caused loss of light perception. ${ }^{23}$ The treated eye of 10 patients was enucleated. In six of them this was due to tumour regrowth that usually occurred during the first postoperative year. The 3 and 5 year probabilities of enucleation were $8 \%$ and $12 \%$, respectively, similar to other irradiated eyes. ${ }^{812-14}$

The goal of irradiation is to destroy all viable tumour and prevent local and systemic relapse. In the present series, the 3 year probabilities of being without local and systemic relapse were $89 \%$ and $92 \%$, respectively for small to medium sized melanomas compared with $61 \%$ and $79 \%$, respectively, for large ones. ${ }^{24} \mathrm{~A}$ low probability of radiation related complications is an additional benefit to the patient. In our opinion, their frequency in the present series is not unduly high. One third of irradiated eyes in the presently reported group of patients had the same or better visual acuity 1 to 2 years after therapy, and one fifth after 3 years. ${ }^{23}$ Ruthenium plaques are a viable alternative to arrest tumour growth, save the eye, and retain a considerable proportion of remaining sight, especially when the tumour is small to medium sized.

The study was supported by grants from the Paulo Foundation and the Eye and Tissue Bank Foundation, Helsinki, Finland.

The participation of Leila Laatikainen, $M D$, in the treatment and follow up of many patients during the early phases of this study is highly appreciated. Furthermore, the help of the Kuopio, Oulu, and Turku University Central Hospitals, as well pio, Oulu, and Turku University Central Hospitals, as well a Jyväskylä Central Hospital

1 Stallard HB. Malignant melanoma of the choroid treated with radioactive applicators. Ann $R$ Coll Surg En $1961 ; 29: 170-82$

2 Stallard HB. Radiotherapy for malignant melanoma of the choroid. Br f Ophthalmol 1966;50:147-55.

3 Lommatzsch PK. Treatment of choroidal melanomas with ${ }^{106} \mathrm{Ru} /{ }^{106} \mathrm{Rh}$ beta-ray applicators. Surv Ophthalmol 1974; 19:85-100.

4 Char DH, Castro JR. Helium ion therapy for choroidal melanoma. Arch Ophthalmol 1982;100:935-8.

5 Gragoudas ES, Seddon J, Goitein M, Verhey L, Monzenrider J, Urie M, et al. Current results of proton beam irradiation of uveal melanomas. Ophthalmology 1985;92:284 91.

6 Lommatzsch PK. $\beta$-irradiation of choroidal melanomas with ${ }^{106} \mathrm{Ru} /{ }^{106} \mathrm{Rh}$ applicators. 16 years' experience. Arch Ophthalmol 1983;101:713-7.

7 Lommatzsch PK. Results after $\beta$-irradiation $\left({ }^{106} \mathrm{Ru} /{ }^{106} \mathrm{Rh}\right)$ of choroidal melanomas: 20 years' experience. $B r f$ Ophthalmol 1986;70:844-51.

8 Char DH, Castro JR, Kroll SM, Irvine AR, Quivey JM, Stone RD. Five-year follow-up of helium ion therapy for uveal melanoma. Arch Ophthalmol 1990;108:209-14.

9 Gragoudas ES, Seddon JM, Egan KM, Glynn RJ, Goitein $\mathrm{M}$, Monzenrider $\mathrm{J}$, et al. Proton irradiation of uveal melanomas: the first 1000 patients. In: Bornfeld $\mathrm{N}$ Gragoudas ES, Höpping W, Lommatzsch PK, Wessing A Zografos L, eds. Tumours of the eye. Amsterdam: Kugler Publications, 1991:489-95.

10 Hillis A. Improving reporting of follow-up data. Am $f \mathrm{Oph}$ thalmol 1982;93:250-3.

11 Seigel D. Analysis of follow-up data. Arch Ophthalmol 1985; 103:647-8.

12 Char DH, Castro JR, Quivey JM, Phillips TL, Irvine AR, Stone $\mathrm{RD}$, et al. Uveal melanoma radiation. ${ }^{125} \mathrm{I}$ brachytherapy versus helium ion irradiation. Ophthalmology 1989; 96:1708-15. 
13 Decker M, Castro JR, Linstadt DE, Char D, Petti PL Quivey JM, et al. Ciliary body melanoma treated with helium particle irradiation. Int $\mathcal{f}$ Radiat Oncol Biol Phys 1990;19:243-7.

14 Lindstadt D, Castro J, Char D, Decker M, Ahn D, Petti P, et al. Long-term results of helium ion irradiation of uveal melanoma. Int f Radiat Oncol Biol Phys 1990;19:613-8.

15 Meecham WJ, Char DH, Kroll S, Catro JR, Blakely EA Anterior segment complications after helium ion radiation therapy for uveal melanoma. Arch Ophthalmol 1994;112 197-203.

16 Guyer DR, Mukai S, Egan KM, Seddon JM, Walsh SM, Gragoudas ES. Radiation maculopathy after proton beam irradiation for choroidal melanoma. Ophthalmology 1992 . 99:1278-85.

17 Kleineidam M, Augsburger J, Hernandez C, Glennon P, Brady LW. Cataractogenesis after cobalt-60 eye plaque
radiotherapy. Int $\mathcal{F}$ Radiat Oncol Biol Phys 1993;26:625-30

18 Quivey JM, Char DH, Phillips TL, Weaver KA, Castro JR Kroll SM. High intensity 125 -iodine $\left({ }^{125} \mathrm{I}\right)$ plaque treatment of uveal melanoma. Int $\mathcal{f}$ Radiat Oncol Biol Phys 1993;26:613-8.

19 Lommatzsch PK, Alberti W, Lommatzsch R, Rohrwacher F. Radiation effects on the optic nerve observed after brachytherapy of choroidal melanomas with ${ }^{106} \mathrm{Ru} /{ }^{106} \mathrm{Rh}$ plaques. therapy of choroidal melanomas with ${ }^{10}{ }^{2} \mathrm{Ru} /{ }^{106} \mathrm{Rh}$ p

20 Char DH, Lonn LI, Margolis LW. Complications of cobalt plaque therapy of choroidal melanomas. Am $\mathcal{f}$ Ophthalmol 1977;84:536-41.

21 Foerster MH, Bornfeld N, Schulz U, Wessing A, MeyerSchwickerath G. Complications of local beta radiation of uveal melanomas. Graefes Arch Clin Exp Ophthalmol 1986;224:336-40.

22 Seddon JM, Gragoudas ES, Egan KM, Glynn RJ, Munzenrider JE, Austin-Seymour $\mathbf{M}$, et al. Uveal melanomas near the optic disc or fovea. Visual results after proton beam irradiation. Ophthalmology 1987;94:354-61.

23 Summanen $P$, Immonen I, Kivelä T, Tommila P, Heikkonen J, Tarkkanen A. Visual outcome of eyes with malignant melanoma of the uvea after ruthenium plaque radiomelanoma of the uvea after ruthenium plaque
therapy. Ophthalmic Surg Lasers 1995;26:449-60.

24 Summanen P, Immonen I, Heikkonen J, Tommila P, Laatikainen L, Tarkkanen A. Survival of patients and metastatic and local recurrent tumour growth in malignant melanoma of the uvea after ruthenium plaque radiotherapy. Ophthalmic Surg 1993;24:82-90.

25 Hermanek P, Sobin LH, eds. TNM classification of malignant tumours, 4th ed. Berlin: Springer Verlag, 1987:158-61.

26 Peto J. The calculation and interpretation of survival curves. In: Buyse ME, Staquet MJ, Sylvester RJ, eds. Cancer clinical trials. Methods and practice. Oxford: Oxford University Priass, 1988:361-80.

27 MacFaul PA, Bedfird MA. Ocular complications after therapeutic irradiation. Br $\mathcal{F}$ Ophthalmol 1970;54:237-47.

28 Brown GC, Shields JA, Sanborn G, Augsburger JJ, Savino Brown GC, Shields JA, Sanborn G, Augsburger JJ, Savino
PJ, Schatz NJ. Radiation retinopathy. Ophthalmology 1982; 89:1494-501.

29 Brown GC, Shields JA, Sanborn GJ, Augsburger J, Savino PJ, Schatz NJ. Radiation optic neuropathy. Ophthalmology 1982;89:1489-93.

30 Dixon WJ, Brown MB, Engleman L, eds. BMDP statistical software. Los Angeles, CA: University of California Press, 1993. 